\title{
Balkanologie
}

Balkanologie Revue d'études pluridisciplinaires

Vol. VII, $n^{\circ} 1$ | 2003

Volume VII Numéro 1

\section{La transition dans les pays balkaniques : les obstacles à la stabilisation macroéconomique}

Le cas de l'Albanie, de la Bulgarie et de la Roumanie

\section{Georges Makris}

\section{OpenEdition}

\section{Journals}

Édition électronique

URL : https://journals.openedition.org/balkanologie/484

DOI : 10.4000/balkanologie.484

ISSN : 1965-0582

\section{Éditeur}

Association française d'études sur les Balkans (Afebalk)

Édition imprimée

Date de publication : 1 juin 2003

Pagination : 189-206

ISSN : 1279-7952

\section{Référence électronique}

Georges Makris, «La transition dans les pays balkaniques : les obstacles à la stabilisation macroéconomique », Balkanologie [En ligne], Vol. VII, n 1 | 2003, mis en ligne le 19 février 2009, consulté le 28 juin 2022. URL : http://journals.openedition.org/balkanologie/484 ; DOI : https://doi.org/ 10.4000/balkanologie.484 


\title{
LA TRANSITION DANS LES PAYS BALKANIQUES : LES OBSTACLES À LA STABILISATION MACROÉCONOMIQUE LE CAS DE L'ALBANIE, DE LA BULGARIE ET DE LA ROUMANIE
}

\author{
Georges MAKRIS*
}

Le terme de transition ne doit pas être considéré comme parfaitement propice, car il sous-entend la convergence - dans une certaine limite temporelle - des systèmes économiques, et plus précisément l'adoption par les économies jusque récemment à planification centrale du modèle des économies de marché développées, d'une façon qui témoigne une conception téléologique de ce processus, comme cela s'est déroulé dans le cas des pays en voie de développement ${ }^{1}$. Peut-être le terme de "transformation" que R. Boyer utilise ${ }^{2}$, ou encore celui de "mutation"3 seraient-ils préférables. Toutefois, dans le cas des pays ex-socialistes de l'Europe centrale et orientale et des Balkans, s'agit-il d'un phénomène très particulier : c'est la première fois qu'une tentative de rationalisation du circuit production - distribution - consommation des biens et services au moyen des mécanismes du marché coïncide avec celle du rétablissement de la démocratie politique. D'où la question sur les possibilités de satisfaire ces aspirations.

Au début des années 9o, le cas de ces pays ne se distinguait pas de celui des pays en voie de développement 4 , ce qui a inspiré les politiques à caractère

\footnotetext{
'Professeur assistant, Département d'Etudes Balkaniques (Florina), Université Aristote de Thessalonique, gmakri@balkan.auth.gr

${ }^{1}$ Chavigny (Régis), " Economies en transition et économies en développement : une comparaison ", Problèmes Economiques, (2563), avril 1998, p. 4.

${ }^{2}$ Boyer (Robert), " La grande transformation de l'Europe de l'Est : Une lecture régulationniste ", Préface à l'édition japonaise de l'ouvrage Yamada (T.), Boyer (Robert), eds., La grande transformation du socialisme, Tokyo : Fujiwara, 1993

${ }^{3}$ Nuti (M.), " Transition ou mutations. Pour une une nouvelle économie politique de la transformation postcommuniste ", Congrès annuel de l'AFSE, Paris, septembre 1996.
}

4 Lavigne (Marie), " Du plan au marché : quelle économie pour le XXIe siècle ? ", Economies et Sociétés, série G (44), avril-mai, p. 24 
monétariste ainsi que l'effort d'application de réformes institutionnelles, qui ont été dictées de manière uniforme par des organismes internationaux (Banque Mondiale, FMI, etc.) sans tenir compte des particularités de l'organisation sociale de ces pays (traits sociaux, économiques, culturels) ou encore des conditions politiques ou même géographiques qui prévalaient dans chaque pays au moment du déclenchement de la transition (les "conditions initiales")5.

Cependant, après plus d'une décennie, l'expérience relative nous permet de nous poser quelques questions. En effet, la poursuite de politiques de stabilisation macroéconomique, en parallèle avec les réformes institutionnelles entreprises dans le but d'opérer le passage à l'économie de marché, et qui ont été souvent liées à l'aide extérieure (par exemple le programme Phare) ${ }^{6}$, n'ont pas déclenché les résultats souhaités. Et si dans certains pays de l'Europe centrale aparaissent des signes d'une certaine reprise, dans d'autres - comme la plupart des pays balkaniques et ceux qui sont issus de l'Union soviétique - la récession persistante continue à créer de fortes inquiétudes. Des phénomènes tels que la baisse du rythme de croissance du PIB par habitant, l'aggravation des disparités de revenus 7 , la détérioration du niveau de vie ${ }^{8}$, ainsi que celle de l'indice du développement humain 9 , prouvent que, souvent, la transition ne se réalise pas "à visage humain"10, et que même son propre processus se trouve compromis. On ne doit pas, bien sûr, oublier que beaucoup de ces pays rencontrent de graves problèmes politiques ou que leur économie est sévèrement affectée par des conflits militaires et des déplacements massifs de populations. Pourtant, l'obstacle le plus important au succès des mesures de stabilisation macroéconomique (et de la politique de croissance) semble être l'uniformité des moyens proposés au moment de la mise en marche de la transition ${ }^{11}$, face à des niveaux de développement et des modèles d'organisation sociale différents.

Dans cet article, nous nous proposons de démontrer la liaison ambivalente existant entre les caractéristiques économiques particulières des pays en transition et la poursuite d'une politique de stabilisation macroéconomique,

5 Cf. Melo (Martha De), Denizer (Cevdet), Gelb (Alan), Tenev (Stoyan), Circumstance and Choice : The role of initial conditions and policies in transition economies, World Bank Policy Research Working Paper (1866), 1997 ; Krueger (Gary), Ciolko (Marek), “ A note on initial conditions and liberalization during transition ", Journal of Comparative Economics, (26), 1998.

${ }^{6}$ Kotios (Aggelos), " La politique balkanique de l'Union européenne ", Marché sans Frontières, 5 (3), 2000.

7 Programme des Nations Unies pour le Développement, La répartition des revenus dans le monde, 1996, p. 30 ; Flemming (John), “Equitable Economic Transformation ", in Tanzi (Vito), Ke-Young (Chu), eds., Income Distribution and High Quality Growth, Cambridge : MIT Press, 1998.

${ }^{8}$ Flemming (John), art.cit.

9 Programme des Nations Unies pour le Développement, op.cit, p. 29.

10 Chavigny (Régis), art.cit., p. 5.

${ }^{11}$ Mordacq (Patrick), " Commentaire ", Problèmes Economiques, (2638-2639), novembre 1999, p. 73. 
par le biais des obstacles que celles-ci présentent à l'application de celle-là, de telle sorte que cette dernière apparaisse quasiment erratique. L'ambivalence de cette liaison consiste en ce que non seulement ces caractéristiques entravent la politique macroéconomique, mais également que cette demière agit sur eux en modifiant leur intensité et en créant ainsi des effets secondaires, et ainsi de suite, selon un cercle vicieux. D'ailleurs, il existe de multiples rapports d'influence mutuelle entre ces caractéristiques, de sorte que dans la plupart des cas elles agissent sur la politique macroéconomique de façon combinée (et inversement). Bien sûr, pour que cette approche soit complète, on aurait dû tenir compte des caractéristiques particulières, sociales, culturelles, politiques, etc., de ces pays, qui peuvent avoir une influence directe ou indirecte sur l'application des réformes et de la politique économique, mais cela dépasserait de beaucoup tant le domaine de l'analyse économique que les limites de ce travail.

Parmi les pays balkaniques, nous avons choisi, pour notre analyse, l'Albanie, la Bulgarie et la Roumanie pour des raisons qui tiennent aussi bien à la disponibilité des données statistiques qu'à l'appartenance de ces pays au même groupe selon les performances en matière de transition ${ }^{12}$.

Quant à la méthodologie que nous allons suivre, il faut souligner que la période examinée est relativement courte et que les lacunes ou différences observées entre les sources statistiques disponibles interdisent l'utilisation d'un modèle économétrique quelconque qui, d'ailleurs, fait défaut dans le cadre de la théorie de la transition. Mais la raison la plus importante du non recours à une méthode économétrique réside dans les particularités des conditions dans lesquelles a été déclenché le processus de "transformation" des institutions économiques de ces pays, particularités qui ne nous permettent pas de définir avec certitude s'il s'agit d'une transformation "autonome" ou "non autonome" : s'agit-il bien de facteurs exogènes qui ont provoqué ce phénomène (changements survenus dans les préférences de la société, dans la technologie au sens "schumpetérien" et dans la répartition des ressources, comme le modèle néoclassique l'admet) ? Même dans le cas où l'on serait à même de répondre à cette question, nous ne sommes pas sûrs de pouvoir expliquer la nature de la réalisation de cette transformation. En d'autres termes, ni le modèle

\footnotetext{
${ }^{12}$ Lenain (Patrick), " Le bilan contrasté de la stabilisation ", Problèmes Economiques, (2638-2639), novembre 1999, pp. 26-28 [extrait de : Dix années de transition - Rapport d'étape, Finances et développement, Fonds Monétaire International, septembre 1998]. Selon ce critère, on peut distinguer deux grands groupes de pays en transition : le premier est constitué des pays qui ont le plus avancé en matière de réformes institutionnelles visant à leur intégration à l'économie de marché et qui présentent des grandeurs macroéconomiques relativement améliorées (pays baltes, Hongrie, Pologne, République tchèque, République slovaque, Slovénie et Croatie), et le second comprend les pays qui en présentent des résultats moins satisfaisants (Albanie, Bulgarie, Roumanie, FYROM, Bosnie-Herzégovine, Yougoslavie et pays provenant de l'ex-URSS).
} 
néoclassique, comparatif et statique, de la "transformation institutionnelle" (Demsetz), ni celui de la "négociation de changements institutionnels" de la nouvelle économie politique (davantage réaliste puisqu'il accepte l'information imparfaite, et peut donc mener à des impasses), ni les modèles de la "théorie des choix publics" (qui tiennent compte du coût des négociations) ou des "économies constitutionnelles" (Buchanan) s'avèrent capables de constituer la base d'une théorie de la transition, bien qu'ils aident à mieux comprendre la complexité du phénomène ${ }^{13}$. Les discussions théoriques sur la transition d'une économie socialiste à une économie de marché ont été centrées primordialement sur le rythme de la transition (thérapie de choc ou masse critique de réformes ?) ou sur les priorités à suivre (création de structures juridiques et sociales nouvelles, stabilisation macroéconomique, ou ouverture au marché international et à la libéralisation des prix ?). Toutefois, ces approches ne semblent pas avoir été exploitées pleinement pour réussir à expliquer comment la transition se réalise. Et cela parce que la transition est un phénomène complexe, pas seulement économique, et qui touche à tous les aspects de l'entité sociale.

L'analyse serait donc de nature empirique, ce qui n'empêche pas cependant d'en tirer des conclusions convaincantes.

\section{LA MORPHOLOGIE DES ÉCONOMIES}

Les économies de tous les pays ex-socialistes en transition depuis la fin des années 80 présentent certains traits communs qui varient d'un pays à l'autre quant à l'ampleur ou l'intensité et dont l'origine doit être recherchée dans la période de l'économie planifiée et / ou au stade précis de développement ou dans leur organisation sociale. Ces caractéristiques, sous l'effet des conditions de réalisation de la transition et sous l'action des mesures de stabilisation macroéconomique, se transforment en des facteurs critiques pour la réussite de cette dernière. En outre, la perspective d'élargissement de l'Union européenne (la Bulgarie et la Roumanie sont concernées depuis 1995) agit comme un catalyseur à plusieurs points de vue.

Les caractéristiques communes les plus importantes sont :

\section{Le dualisme du tissu productif}

Ce dualisme apparaît sous plusieurs formes : il s'agit, d'abord, de la coexistence d'un secteur étatique, qui reste encore très important, d'un côté, et

${ }^{13}$ Cf. Wagenex (Hans-Jurgen), " La transformation : un cadre historique et théorique ", Revue d'études comparatives Est-Ouest, 29 (4), décembre 1998. 
d'un secteur privé croissant, qui présente de nombreuses singularités, de l'autre. Plus spécifiquement, au sein de ce dernier, on rencontre de grandes inégalités entre une minorité de branches qui reçoivent des investissements étrangers directs et les autres dont l'activité est exclusivement basée sur des ressources internes. L'autre caractéristique est la coexistence d'un grand nombre de petites entreprises - qui appartiennent souvent au secteur "atypique"14 - et de grandes entreprises étatiques héritées du passé et qui rencontrent des difficultés face à leur privatisation et à leur modernisation. Comme on peut le constater à partir des données issues du Transition Report 2000 de la BERD, l'Albanie semble avoir réalisé l'indice de privatisation des petites entreprises le plus élevé et la participation du secteur privé au PIB a évolué le plus rapidement atteignant $75 \%$ en fin de période, alors que la Bulgarie a opté pour la privatisation des grandes entreprises ainsi que pour les réformes visant à stimuler l'initiative d'entreprise (participation du secteur privé au PIB en 1999 : $70 \%$ ). Les performances de la Roumanie en la matière ne peuvent être caractérisées que comme médiocres (participation du secteur privé au PIB en 1999 : 60\%). En ce qui concerne les nouvelles petites entreprises, on constate une diminution du rythme de leur création. Les causes avancées pour l'expliquer sont la difficulté à se procurer le capital nécessaire, l'imposition encore élevée, la bureaucratie et l'absence d'encadrement des initiatives d'entreprise par des institutions spécialisées ${ }^{15}$.

Quant à la structure industrielle de ces pays, elle reste, en règle générale, typiquement celle de pays à avantages comparatifs statiques et est largement liée à des conditions de production de main d'oeuvre bon marché, de matières premières et de grande consommation d'énergie ${ }^{16}$.

Dans de telles conditions, l'application des réformes destinées surtout à la libéralisation du commerce extérieur et des prix ne semble pas avoir contribué à restructurer de façon substantielle le système productif, même si l'on ne tient pas compte du premier choc de la transition, d'ailleurs considéré comme attendu. On pourrait, au contraire, parler d'effets pervers qui constituent des entraves à la poursuite d'une politique économique contraignante, impliquant l'intervention des pouvoirs publics afin de maintenir la cohérence sociale.

Parallèlement, il faut tenir compte de l'économie souterraine qui reste encore aujourd'hui une part importante de l'activité économique (elle a été

14 Il s'agit d'activités nécessitant peu de capital, protégées de la concurrence internationale et ne fonctionnant pas selon des critères purement capitalistiques (Bureau International du Travail, Rapport Général de la $14^{e}$ conférence internationale des statistiques du travail, 1987, ch. 2, pp. 9-27).

${ }_{15}$ Pour plus de détails, voir Le retard des entreprises privées, synthèses de l'OCDE (avril 1999).

${ }^{16}$ Totev (Stoyan Anastassov), " Economic performance and structure of southeastern european countries ", in Kotios (Aggelos), Petrakos (George), Restructuring and Development in Southeastern Europe, Volos : University of Thessaly Press, Volos, 2002. 
qualifiée comme seule expression de l'économie marchande durant la période de planification centrale). On estime qu'en 1995 l'économie souterraine représentait $50 \%$ du nouveau secteur privé. La différence résulte du fait que son intégration à l'économie officielle est d'autant plus facile que les réformes institutionnelles sont avancées ${ }^{17}$.

\section{La part élevée du secteur agricole et le processus de désindustrialisation}

Le secteur agricole traditionnel reste encore très élevé dans le PIB de ces pays ; sa part, selon les services de statistiques nationales étant en 1999 de $17,3 \%$ pour la Bulgarie, $13,9 \%$ pour la Roumanie et $52,5 \%$ pour l'Albanie (en 1990 , elle était de $37,9 \%$, dû surtout au déclin du secteur industriel), alors qu'il semble absorber de plus en plus de main-d'œuvre (entre 1990 et 1999 l'occupation dans ce secteur a augmenté selon les mêmes sources de $36,5 \%$ pour la Bulgarie et de 50,6\% pour l'Albanie).

Quant au phénomène du déclin industriel, il est considéré comme résultant de l'incapacité de réponse qualitative de l'offre à la demande et de la déformation des structures productives due à l'épargne forcée ainsi qu'au mode de gestion des ressources durant la période de planification centrale de ces économies. Plus précisément, le taux d'investissement dans le PIB était, avant la transition, d'au moins $25 \%$ plus élevé que dans les économies de marché, ce qui reflète la négligence des méthodes d'organisation et des systèmes de motivation de la part des planificateurs. Ceci a eu comme conséquence la faible productivité du capital et du travail, ainsi que de l'ICOR (Incremental CapitalOutput Ratio $)^{18}$. En ce qui concerne les produits manufacturés, ils sont en règle générale incapables de concurrencer ceux des pays industrialisés de l'Europe, mais parviennent à satisfaire une partie au moins de la demande interne, qui commence à se différencier sous l'influence de la libéralisation du commerce extérieur et de l'accentuation de la disparité des revenus. Toutefois, les effets des réformes sur l'industrie de ces pays ne semblent pas, pour l'instant, être encourageants, nécessitant le soutien des gouvernements : comme on peut le constater à partir des tableaux 1 et 2, tant la production que l'emploi dans le secteur industriel connaissent une baisse soutenue, surtout dans le cas de la Bulgarie et de la Roumanie.

${ }^{17}$ Mordacq (Patrick), art.cit., p. 70.

${ }^{18} \mathrm{Götz}$ (Robert), “ Le modèle économique socialiste à la veille de la transition ", Problèmes Economiques, (2638-2639), novembre 1999, p. 12. 
Tableau 1 : La production industrielle en \% du PIB, 1991-1999

\begin{tabular}{|l|c|c|c|c|c|c|c|c|c|}
\hline & 1991 & 1992 & 1993 & 1994 & 1995 & 1996 & 1997 & 1998 & 1999 \\
\hline Albanie & - & 16,9 & 13,9 & 12,5 & 11,7 & 12,2 & 12,4 & 11,9 & 11,9 \\
\hline Bulgarie & - & 39,0 & 32,7 & 29,9 & 31,0 & 28,5 & 25,3 & 25,5 & 24,6 \\
\hline Roumanie & - & 38,3 & 33,8 & 36,2 & 32,9 & 34,2 & 35,6 & 27,5 & 27,8 \\
\hline
\end{tabular}

Source : BERD, Transition report 2000.

Tableau 2 : L'emploi dans le secteur industriel en \% de l'ensemble, 1991-1999

\begin{tabular}{|l|c|c|c|c|c|c|c|c|c|}
\hline & 1991 & 1992 & 1993 & 1994 & 1995 & 1996 & 1997 & 1998 & 1999 \\
\hline Albanie & - & - & - & 10,2 & 8,3 & 7,6 & 8,0 & 7,8 & - \\
\hline Bulgarie & 34,5 & 32,6 & 30,4 & 29,1 & 28,1 & 27,5 & 26,6 & 24,7 & 23,0 \\
\hline Roumanie & 35,3 & 31,6 & 30,1 & 26,4 & 24,3 & 25,1 & 22,2 & 22,0 & - \\
\hline
\end{tabular}

Source : BERD, Transition report 2000.

\section{Le sous-développement du secteur financier et bancaire}

Le marché financier et toutes les institutions financières en général ont dû être créés pour la première fois. Pendant les premières années de la transition, on observe la création d'un grand nombre de banques de petite taille aux exigences de statut floues et dont le contrôle étatique laisse à désirer, ce qui a eu comme résultat nombre de faillites ou de fusions. En même temps, l'effort d'assainissement et de privatisation des grandes banques étatiques, qui reçoivent la majorité des dépôts, a été rendu difficile à cause notamment des prêts octroyés sans respect des critères économiques dans le passé ou liés à des garanties douteuses pendant les premières années de la transition. D'ailleurs, la politique de refinancement que les banques centrales ont suivi surtout en Bulgarie (jusqu'en 1997, année de la mise en place d'un "currency board" et l'alignement du lev au deutschmark) et en Roumanie a largement contribué à l'instabilité monétaire constituant ainsi une entrave rédhibitoire à la stabilité de la croissance. Progressivement, des règles plus rigoureuses ont été adoptées à l'égard du système bancaire, dont d'ailleurs le statut se trouve amélioré du fait de la concurrence exercée par les banques étrangères venues s'installer à un rythme croissant (surtout en Bulgarie et en Roumanie). Toutefois, l'insuffisance de l'épargne locale et la méfiance du public face au système bancaire - il en résulte de très faibles multiplicateurs monétaires - en combinaison avec l'âge embryonnaire des autres institutions financières, surtout de la bourse des valeurs et du marché des assurances, ainsi que le retard pris par la législa- 
tion pour assurer la transparence des transactions, ne permettent pas encore le développement du secteur bancaire au niveau que nécessite l'effort de stabilisation et de croissance économiques ${ }^{19}$ (tableau 3 ).

\section{Tableau 3 : Secteur bancaire et financier, 1991-2000}

\begin{tabular}{|l|c|c|c|c|c|c|c|c|c|c|}
\hline Albanie & 1991 & 1992 & 1993 & 1994 & 1995 & 1996 & 1997 & 1998 & 1999 & 2000 \\
\hline $\begin{array}{l}\text { Nombre de banques } \\
\text { (dont étrangères) }\end{array}$ & - & - & - & $6(3)$ & $6(3)$ & $8(3)$ & $9(3)$ & - & - & - \\
\hline $\begin{array}{l}\text { Dépôts auprès des } \\
\text { banques de l'Etat } \\
\text { (en\% de l'ensemble) }\end{array}$ & - & - & - & 97,8 & 94,5 & 93,7 & 89,9 & - & - & - \\
\hline $\begin{array}{l}\text { Prêts douteux } \\
\text { (en\% de l'ensemble) }\end{array}$ & - & - & - & - & 34,9 & 40,1 & 93,1 & - & - & - \\
\hline $\begin{array}{l}\text { Prêts domestiques } \\
\text { aux entreprises } \\
\text { (en \% du PIB) }\end{array}$ & - & - & - & 3,9 & 3,6 & 3,9 & 3,8 & 3,2 & 3,6 & - \\
\hline $\begin{array}{l}\text { Capitalisation des } \\
\text { titres (en \% du PIB) }\end{array}$ & - & - & - & - & - & - & - & - & - & - \\
\hline $\begin{array}{l}\text { Indice de la BERD pour } \\
\text { les réformes du } \\
\text { secteur bancaire }\end{array}$ & 1,0 & 1,0 & 1,3 & 2,0 & 2,0 & 2,0 & 2,0 & 2,0 & 2,0 & - \\
\hline $\begin{array}{l}\text { Indice de la BERD pour } \\
\text { les réformes du } \\
\text { secteur non bancaire }\end{array}$ & 1,0 & 1,0 & 1,0 & 1,0 & 1,0 & 1,7 & 1,7 & 1,7 & 1,7 & - \\
\hline
\end{tabular}

\begin{tabular}{|l|c|c|c|c|c|c|c|c|c|c|}
\hline Bulgarie & 1991 & 1992 & 1993 & 1994 & 1995 & 1996 & 1997 & 1998 & 1999 & 2000 \\
\hline $\begin{array}{l}\text { Nombre de banques } \\
\text { (dont étrangères) }\end{array}$ & $75(0)$ & $79(0)$ & $41(0)$ & $40(0)$ & $41(3)$ & $42(3)$ & $28(7)$ & $34(17)$ & $34(22)$ & $35(25)$ \\
\hline $\begin{array}{l}\text { Dépôts auprès des } \\
\text { banques de l'Etat } \\
\text { (en \% de l'ensemble) }\end{array}$ & - & - & - & - & - & 82,2 & 66,0 & 56,4 & 50,5 & 19,8 \\
\hline $\begin{array}{l}\text { Prêts douteux } \\
\text { (en \% de l'ensemble) }\end{array}$ & - & - & 6,6 & 6,8 & 12,6 & 14,6 & 12,9 & 11,8 & 17,5 & 10,9 \\
\hline $\begin{array}{l}\text { Prêts domestiques } \\
\text { aux entreprises } \\
\text { (en \% du PIB) }\end{array}$ & 7,2 & 5,8 & 3,7 & 3,8 & 21,1 & 35,6 & 12,6 & 12,7 & 14,6 & - \\
\hline $\begin{array}{l}\text { Capitalisation des } \\
\text { titres (en \% du PIB) }\end{array}$ & - & - & - & - & 0,5 & 0,2 & 0,0 & 7,7 & 6,0 & - \\
\hline $\begin{array}{l}\text { Indice de la BERD pour } \\
\text { les réformes du } \\
\text { secteur bancaire }\end{array}$ & 1,0 & 1,7 & 2,0 & 2,0 & 2,0 & 2,0 & 2,7 & 2,7 & 2,7 & - \\
\hline $\begin{array}{l}\text { Indice de la BERD pour } \\
\text { les réformes du } \\
\text { secteur non bancaire }\end{array}$ & 1,0 & 1,0 & 1,0 & 1,0 & 2,0 & 2,0 & 2,0 & 2,0 & 2,0 & - \\
\hline
\end{tabular}

$19 \mathrm{Cf}$. Exeter (J.), Fries (Steven), Le processus de transition postcommuniste-Modalités et perspectives, FMI : Finances et Développement, septembre 1998. 


\begin{tabular}{|l|c|c|c|c|c|c|c|c|c|c|}
\hline Roumanie & 1991 & 1992 & 1993 & 1994 & 1995 & 1996 & 1997 & 1998 & 1999 & 2000 \\
\hline $\begin{array}{l}\text { Nombre de banques } \\
\text { (dont étrangères) }\end{array}$ & - & - & - & $20(3)$ & $24(6)$ & $31(8)$ & $31(13)$ & $36(16)$ & $34(19)$ & 33 (21) \\
\hline $\begin{array}{l}\text { Dépóts auprès des } \\
\text { banques de l'Etat } \\
\text { (en \% de l'ensemble) }\end{array}$ & - & - & - & 80,4 & 84,3 & 80,9 & 80,0 & 75,3 & 50,3 & 45,0 \\
\hline $\begin{array}{l}\text { Prêts douteux } \\
\text { (en \% de l'ensemble) }\end{array}$ & - & - & - & 18,5 & 37,9 & 48,0 & 56,5 & 67,5 & 36,6 & - \\
\hline $\begin{array}{l}\text { Prêts domestiques } \\
\text { aux entreprises } \\
\text { (en \% du PIB) }\end{array}$ & - & - & - & - & - & 11,5 & 13,7 & 15,2 & 10,5 & - \\
\hline $\begin{array}{l}\text { Capitalisation des } \\
\text { titres (en\% du PIB) }\end{array}$ & - & - & - & 0,0 & 0,4 & 0,2 & 2,0 & 3,0 & 3,1 & - \\
\hline $\begin{array}{l}\text { Indice de la BERD pour } \\
\text { les réformes du } \\
\text { secteur bancaire }\end{array}$ & 1,0 & 1,0 & 1,0 & 2,0 & 3,0 & 3,0 & 2,7 & 2,3 & 2,7 & - \\
\hline $\begin{array}{l}\text { Indice de la BERD pour } \\
\text { les réformes du } \\
\text { secteur non bancaire }\end{array}$ & 1,0 & 1,0 & 1,0 & 2,0 & 2,0 & 2,0 & 2,0 & 2,0 & 2,0 & - \\
\hline
\end{tabular}

Source : BERD, Transition report 2000 ; FMI, WIIW, BNB, BNR.

\section{L'intégration dans l'économie internationale}

Les économies des pays ex-socialistes de l'Europe centrale et orientale fonctionnaient protégées de toute concurrence dans le cadre de la répartition internationale du travail du COMECON. L'effondrement de ces rapports réciproques, l'ouverture presque totale au commerce mondial, et surtout avec l'UE, constituent pour eux une épreuve difficile : l'affrontement avec les prix mondiaux et le retard de leur offre par rapport aux normes internationales ont provoqué au début des années 90 une diminution drastique du volume de leur commerce extérieur. Cela a bien sûr contribué au rétablissement d'un certain niveau de concurrence, mais aussi à l'adaptation des prix aux niveaux internationaux, ce qui a eu comme effet la hausse des pressions inflationnistes et la détérioration des termes du commerce. Et alors que certains produits pour lesquels les pays balkaniques possèdent des avantages comparatifs continuent à être soumis à des limitations quantitatives et d'autres restrictions ${ }^{20}$, la part de l'UE à leur commerce extérieur a doublé jusqu'en 1995. Depuis, cette évolution fléchit (à cela il faut ajouter la chute libre de leurs exportations vers la Russie, l'Ukraine et la Biélorussie, conséquence de la crise monétaire russe de 1998), en parallèle avec la persistance, voire l'aggravation, de leurs déficits commerciaux (et surtout des transactions courantes). Si ce phénomène était accompagné par une hausse de la productivité et par des rythmes positifs de croissance, il n'y aurait pas eu de raisons à s'inquiéter. Mais cela n'a pas eu lieu, et de plus à ces

${ }^{20}$ Lhomel (Edith), “ Albanie 1998-1999 : Aux limites de l'implosion ”, Courrier des pays de l'Est (Le), (442), 1999, p. 16. 
déficits il faut ajouter les déficits publics, ce qui a donné lieu à de forts déséquilibres macroéconomiques vers la fin de la décennie - surtout en Roumanie -, éprouvant fortement la poursuite de leur politique de stabilisation budgétaire et monétaire.

De récentes recherches concernant la relation entre la libéralisation du commerce extérieur et la croissance viennent mettre en doute l'existence d'un rapport positif indéniable. Ce rapport semble être plutôt aléatoire et dépendre d'un certain nombre de facteurs aussi bien internes qu'externes ${ }^{21}$. En ce qui concerne la théorie contemporaine de la croissance endogène, elle accepte que la réponse dépend de l'avantage comparatif du pays en question, c'est-à-dire de sa capacité à endiguer les ressources internes vers des activités garantissant une croissance à long terme ou de les en détourner. Le rapport entre la libéralisation du mouvement des capitaux et la croissance apparaît encore moins clair ${ }^{22}$.

\section{L'ÉVOLUTION DES RÉFORMES}

La Banque Européenne pour la Reconstruction et le Développement (BERD) a élaboré un "indice de transition" exprimant une moyenne des estimations quant aux résultats des réformes entreprises par pays entre 1991 et 1999. Ces réformes concernent les privatisations, les restructurations des entreprises, la libéralisation des prix et du commerce, l'introduction d'une politique pour la concurrence, la réforme du système bancaire et le développement des marchés des valeurs. Sur la base de ces indices (allant de 1 à 4), on peut distinguer six groupes de pays, classés par ordre de moindre performance : les pays de l'Europe centrale et orientale, les pays baltiques, les pays du Sud-Est de l'Europe (l'Albanie, la Bulgarie et la Roumanie y appartiennent, pays pour lesquels nous avons calculé ces indices comme étant 2,6, 2,8 et 2,7 respectivement), les pays du centre de la CIS, les pays du Caucase et les pays de l'Asie Centrale. S'agissant des pays qui nous intéressent, le plus avancé dans les réformes est la Bulgarie (meilleures performances au niveau des privatisations de grande échelle), suivie par la Roumanie (qui devance les autres en matière de libéralisation du commerce et d'application de la politique de concurrence) et l'Albanie. Dans ces trois pays, on observe une accélération des réformes depuis 1997, probablement après recommandation du Fonds Monétaire

${ }^{21} \mathrm{Cf}$. voir Mink (Georges), Szurek (Jean-Charles), "L’ancienne élite communiste en Europe centrale - stratégies, ressources et reconstructions identitaires ", Revue Française de Science Politique, 48 (1), février 1998.

${ }^{22}$ La rédaction, " Entreprises et privatisations en chiffres ", Problèmes Economiques, (2638-2639), novembre 1999, p. 39. 
International et de la Banque Mondiale, qui lient les programmes de financement au progrès des réformes.

Cette distribution géographique des pays en transition, selon le critère du progrès dans les réformes, coïncide en règle générale avec l'évolution des grandeurs macroéconomiques pour la même période. Il aurait été donc aisé d'établir une corrélation positive élevée entre ces deux phénomènes, comme cela apparaît souvent dans la bibliographie internationale. Mais, peut-être est-il possible d'expliquer cette corrélation tant par le niveau de développement de ces économies que par la capacité politique d'appliquer ces réformes, laquelle dépend à son tour de l'organisation sociale de ces pays durant la période de planification centrale ${ }^{23}$. Par exemple, un des facteurs qui peut expliquer les différences dans le retard quantitatif et qualificatif des privatisations des petites et grandes entreprises étatiques est les conditions qui permettent aux managers du régime précédant de conserver leur poste et de développer des stratégies de maximisation de leur profit personnel ${ }^{24}$ dans le cadre de la méthode MEBO (Management and Employee Buy Out), méthode observée particulièrement en Bulgarie. Le refus de nombreuses entreprises étatiques de rembourser leurs dettes envers l'Etat en constitue un autre exemple, comme en Roumanie $^{25}$ (en 1988 cela représentait $24,4 \%$ des ressources publiques), ou le cas de ces entreprises privées accumulant les arriérés de paiement envers les banques (dans le cadre d'une "culture de non-paiement"). D'ailleurs, l'évasion fiscale (qui représente $16 \%$ du PIB en Albanie) entrave de beaucoup le rétablissement de l'équilibre des finances publiques ${ }^{26}$. Tout cela crée des entraves à l'assainissement du secteur bancaire et ne lui permet pas de se développer ni d'accompagner les efforts de la politique économique. On peut encore mentionner le rôle moins important par rapport aux pays de l'Europe centrale (surtout en Hongrie et en Pologne) que joue la "nomenklatura" du régime précédent en train de se transforner en une classe de managers, facteur considérable et controversé de croissance du secteur privé27.

Contrairement à ce qu'on pourrait s'attendre, il semble qu'on ne peut établir de relation directe entre le progrès réalisé dans le domaine des réformes et les investissements directs étrangers (IDE). Cette constatation peut être véri-

\footnotetext{
${ }^{23}$ Kotios (Aggelos), art.cit., p. 172.

${ }^{24}$ Rodrik (Dani), " Les mirages de l'ouverture extérieure ”, Economie Politique, (10), 2001, pp. 49-51.

25 Grilli (Vittorio), Miles-Ferretti (Gian-Maria), " Economic effects and Structural Determinants of Capital Controls ", IMF Staff Papers, 42 (3), september 1995.
}

${ }^{26}$ Wolf (M.), " Le piège de la transition: le comment et le pourquoi d'une évolution en U ", Problèmes Economiques, (2638-2639), novembre 1999 [traduction de "Caught in the transition trap ", Financial Times, $30 / 06 / 99]$.

27 Voir Labaronne (Daniel), “ Les lenteurs de la privatisation de l'Est : une conséquence de la stratégie d'enracinement des managers ", Revue d'Economie Politique, (5), septembre-octobre 1998. 
fiée à partir d'une comparaison de données provenant du FMI, des Banques centrales des pays concernés et de la BERD ${ }^{28}$ : les indices évaluant le progrès des réformes et ceux évaluant les investissements directs étrangers cumulés par habitant ne révèlent pas de corrélation directe. En effet, l'Albanie a reçu un volume d'investissements inférieur à celui de la Bulgarie et, surtout, celui de la Roumanie, par rapport au niveau des réformes réalisées (tableaux 4 et 5). Les réformes ne semblent pas être la seule condition pour attirer les IDE. Le niveau général de développement ainsi que les perspectives d'élargissement de l'UE doivent y jouer un rôle important.

Tableau 4 : Investissements étrangers directs nets, 1991-1999 (millions de US\$)

\begin{tabular}{|l|c|c|c|c|c|c|c|c|c|}
\hline & 1991 & 1992 & 1993 & 1994 & 1995 & 1996 & 1997 & 1998 & 1999 \\
\hline Albanie & 20 & 45 & 65 & 89 & 97 & 42 & 45 & 51 & 92 \\
Bulgarie & 42 & 40 & 105 & 98 & 138 & 507 & 537 & 806 & 500 \\
\hline Roumanie & 73 & 87 & 341 & 417 & 415 & 1.267 & 2.079 & 949 & 500 \\
\hline
\end{tabular}

Source : BERD, Transition report 2000.

Tableau 5 : Réformes et investissements directs étrangers, 1991-1999

\begin{tabular}{|l|c|c|}
\hline & $\begin{array}{c}\text { Indice de la BERD } \\
\text { pour le progrès des réformes }\end{array}$ & $\begin{array}{c}\text { Investissements directs étrangers } \\
\text { cumulés par tête* }\end{array}$ \\
\hline Albanillions de US\$)
\end{tabular}

"La population est prise comme la moyenne de la période 1991-1998.

Source : calculs à partir de données de la BERD, Transition report 2000.

${ }^{28}$ Lhomel (Edith), " Roumanie 1998-1999 : sur le fil du rasoir ", Courrier des pays de l'Est, (442), 1999 , p. 117. 


\section{LES EFFORTS DE STABILISATION MACROÉCONOMIQUE}

L'évolution des grandeurs macroéconomiques prouve clairement que l'attendue "récession de la transformation", qui a été observée pendant les premières années de la transition, a évolué en une "récession structurale". En effet, après la crise du début de la décennie (fortes baisses du PIB et des investissements, augmentation des déficits des balances commerciale et courante, hyperinflation et hausse du chômage), des déséquilibres macroéconomiques réapparaissent autour de 1997 et, depuis, il n'y a pas de signes de reprise durable et surtout d'assainissement de la structure économique : faible hausse ou même baisse du PIB / tête, accentuation des inégalités des revenus (en Roumanie le coefficient Gini a doublé entre 1991 et 1997), la part du secteur industriel dans le produit national diminue rapidement, le chômage atteint des niveaux difficilement supportables, l'inflation - bien que loin des taux du début de la transition - reste, dans la plupart des cas, élevée, les déficits de la balance commerciale et de la balance des opérations courantes se creusent davantage (moins dans le cas de l'Albanie grâce à l'apport des travailleurs à l'étranger), alors que la composition du commerce avec les pays de l'OCDE (surtout avec l'UE) se modifie en faveur de ces derniers, le commerce dans le cadre de l'ex-COMECON s'étant effondré.

Dans ce contexte, si l'on juge la politique économique - fortement erratique jusqu'en 1996-1997 (stop and go policy) - à partir de l'évolution des déficits ainsi que des variables monétaires, on peut constater qu'elle devient davantage rigoureuse et restrictive, bien qu'interrompue par de courtes périodes d'expansion ou caractérisée par des contradictions. Cependant, les données concernant le financement du secteur public montrent un accroissement de sa dépendance des sources internes et externes, alors qu'il devient incapable de financer les interventions nécessaires pour atténuer les entraves à la poursuite de la politique économique.

Si les déséquilibres macroéconomiques des premières années de la transition peuvent être interprétés comme le résultat de la brusque application des réformes sur un ensemble d'institutions peu développées et fragiles, ceux de la fin de la décennie ne peuvent pas être expliqués uniquement par le même schéma. Par exemple, la contraction de l'activité, l'hyperinflation et la hausse du chômage de la première période ont leur origine dans la brusque introduction des réformes (surtout la libéralisation des prix, l'ouverture du commerce extérieur et la rationalisation du fonctionnement des structures productives ${ }^{29}$ des secteurs placés dans le cadre de l'économie du marché - restructuration schumpetérienne). Mais l'inflation persistante des dernières années semble

\footnotetext{
${ }^{29}$ Andreff (Wladimir), "Facteurs inertiels et effet de sentier dans l'analyse théorique de la transition ", Congrès Annuel de l'AFSE, Paris, septembre 1996.
} 
avoir une cause supplémentaire : "l'inflation d'inertie"30, c'est-à-dire provoquée par des causes sociales et politiques, et plus spécialement par l'antagonisme des groupes se disputant des parts du revenu national. Cet antagonisme trouve son origine dans un contexte où la libéralisation des prix, le mode de réalisation des privatisations, le développement du secteur privé, l'économie souterraine et l'intégration à l'économie mondiale, en combinaison avec les politiques de stabilisation fonctionnent dans un cercle vicieux et constituent des facteurs de modification du partage des revenus et de la richesse, de hausse du chômage et donc de création d'impasses à l'exercice de la politique économique même. En d'autres termes, il s'agit des effets ambivalents de l'interaction entre les caractéristiques économiques, les réformes et la politique de stabilisation et de croissance. Elle se déroule de la façon suivante : les caractéristiques propres aux économies de ces pays, qui soit existaient déjà soit se sont manifestées au déclenchement de la transition, exercent sous l'action des réformes des effets combinés tant entre eux que sur les variables macroéconomiques dont le résultat est la création de contraintes économiques, sociales et politiques à l'application d'une politique de rétablissement des déséquilibres macroéconomiques, politique qui à son tour génère des effets négatifs sur ces caractéristiques mêmes.

L'évolution du PIB réel par habitant n'est certes pas positive, mais cela devient encore plus inquiétant avec l'accentuation des disparités des revenus. Les salaires réels suivant une tendance à la baisse - résultat du market clearing ${ }^{31}$ et n'étant pas indexés sur les prix, le coefficient Gini prend des valeurs de plus en plus élevées. Etant donné que le chômage augmente et que les institutions de protection sociale sont peu développées, il devient aisé de conclure que de fortes pressions sociales, et donc politiques, sont exercées vers une politique économique plus expansionniste. Ce qui a comme conséquence l'aggravation des déficits budgétaires, le recours à la Banque centrale et d'autres mesures monétaires "accommodantes" (par exemple baisse des taux d'intérêt à l'emprunt ou augmentation de la masse monétaire), impliquant ainsi une augmentation du volume de la dette publique et de l'endettement extérieur et exerçant des pressions sur les réserves. Outre que le danger d'une nouvelle flambée de l'inflation (surliquidité) devient imminent, l'évolution des taux de change (forte volatilité durant tout le long de la période) reflète les faiblesses de la structure économique et crée des pressions permanentes sur le niveau des prix, compromettant sérieusement l'efficacité de la politique macroéconomique. Face à la diminution du niveau de la production et de la détérioration 
des termes du commerce extérieur qui s'en suivent, il devient difficile de poursuivre une politique de stabilisation et encore moins de croissance permanente. De telles périodes ont été observées dans les trois pays examinés, des premières années de la transition et jusqu'en 1993 et de nouveau en 1996-1997.

Pour pallier une telle évolution, une politique économique de contraction a été instaurée (Albanie en 1997, Bulgarie en 1998, Roumanie en 1999) : diminution des dépenses publiques, hausse des taux d'intérêt à l'emprunt, diminution de la circulation monétaire et des crédits à l'économie. Toutes ces mesures ont été prises selon un ordre différent et selon les possibilités et contraintes de chaque pays. Dans le même temps des mesures contradictoires sont adoptées, comme par exemple en 1995 en Roumanie, où la contraction budgétaire a été accompagnée par une politique monétaire expansionniste (c'est le contraire qui s'est produit en Albanie en 1998-2000).

Comme on peut le constater à partir du tableau 6, l'effort de stabilisation même incomplet est évident, mais le PIB et les autres indicateurs macroéconomiques enregistrent de nouveaux reculs. Dans un tel contexte, toutes les caractéristiques spécifiques des économies étudiées se trouvent à leur tour affectées, engendrant de nouveau leurs effets contraignant sur la politique macroéconomique : le dualisme de l'appareil productif continue à persister malgré l'élargissement de la part du secteur privé dans le PIB (la privatisation des petites entreprises devançant de beaucoup celle des grandes), la désindustrialisation et la part dominante du secteur agricole sont encore plus marquées qu'au début de la transition, la fragilité et le dysfonctionnement du système bancaire et du secteur financier en général n'inspirent toujours pas la confiance du public ni n'attirent non plus les capitaux étrangers (voir tableaux $5 \mathrm{~A}$ et $5 \mathrm{~B}$ ), malgré l'avance des réformes (à un rythme pourtant moins soutenu qu'au début de la transition selon la BERD). Quant au commerce international, les tendances enregistrées déjà au milieu des années 90 ne semblent pas s'inverser. 


\section{Tableau 6 : Politiques et principaux indicateurs macroéconomiques, 1991-2000}

\begin{tabular}{|c|c|c|c|c|c|c|c|c|c|c|}
\hline Politique économique & 1991 & 1992 & 1993 & 1994 & 1995 & 1996 & 1997 & 1998 & 1999 & 2000 \\
\hline $\begin{array}{l}\text { Politique budgétaire } \\
\text { (en \% du PIB) }\end{array}$ & \multicolumn{5}{|c|}{ restriction } & exp. & rest. & \multicolumn{3}{|c|}{ expansion } \\
\hline $\begin{array}{l}\text { Dette publique } \\
\text { (en \% du PIB) }\end{array}$ & - & - & - & - & - & - & - & - & \multicolumn{2}{|c|}{ expansion } \\
\hline $\begin{array}{l}\text { Crédits à l'économie } \\
\text { (évolution annuelle en \%) }\end{array}$ & \multicolumn{3}{|c|}{ expansion } & \multicolumn{2}{|c|}{ restriction } & \multicolumn{2}{|c|}{ expansion } & \multicolumn{3}{|c|}{ restriction } \\
\hline $\begin{array}{l}\text { Taux d'intérêt à l'emprunt } \\
(1 \text { an })\end{array}$ & - & \multicolumn{3}{|c|}{ baisse } & \multicolumn{3}{|c|}{ hausse } & \multicolumn{2}{|c|}{ stabilisation } & - \\
\hline$M_{3}$ & - & \multicolumn{3}{|c|}{ restriction } & \multicolumn{3}{|c|}{ expansion } & rest. & exp. & - \\
\hline $\begin{array}{l}\text { Dette externe } \\
\text { (en \% du PIB) }\end{array}$ & \multicolumn{2}{|c|}{ expansion } & \multicolumn{4}{|c|}{ restriction } & exp. & \multicolumn{2}{|c|}{ restriction } & - \\
\hline $\begin{array}{l}\text { Taux de change } \\
\text { (monn. nat./US\$) } \\
\text { (moyenne annuelle) }\end{array}$ & - & \multicolumn{2}{|c|}{ hausse } & \multicolumn{2}{|c|}{ baisse } & \multicolumn{2}{|r|}{ hausse } & & b. & h. \\
\hline \multicolumn{11}{|l|}{$\begin{array}{l}\text { Indicateurs } \\
\text { macroéconomiques }\end{array}$} \\
\hline $\begin{array}{l}\text { PIB réel/tête } \\
\text { (évolution annuelle en \%) }\end{array}$ & \multicolumn{2}{|c|}{ baisse } & \multicolumn{4}{|c|}{ hausse } & b. & \multicolumn{3}{|c|}{$\begin{array}{c}\text { hausse et } \\
\text { stabilisation }\end{array}$} \\
\hline $\begin{array}{l}\text { Investissements bruts } \\
\text { (en \% du PIB) }\end{array}$ & \multicolumn{2}{|c|}{ stabilisation } & \multicolumn{8}{|c|}{ hausse et stabilisation } \\
\hline Chômage & \multicolumn{3}{|c|}{ hausse } & \multicolumn{3}{|c|}{ baisse } & \multicolumn{3}{|c|}{ hausse } & - \\
\hline Inflation & \multicolumn{2}{|c|}{ forte hausse } & \multicolumn{3}{|c|}{ baisse } & hau & Isse & \multicolumn{3}{|c|}{ baisse et stab. } \\
\hline $\begin{array}{l}\text { Déficit de la balance } \\
\text { commerciale } \\
\text { (en } \% \text { du PIB) }\end{array}$ & \multicolumn{2}{|c|}{ hausse } & \multicolumn{3}{|c|}{ baisse } & h. & & \multicolumn{3}{|c|}{$\begin{array}{c}\text { baisse et } \\
\text { stabilisation }\end{array}$} \\
\hline
\end{tabular}

\section{Bulgarie}

\begin{tabular}{|c|c|c|c|c|c|c|c|c|c|}
\hline Politique économique & 1991 & 1992 & 1993 & 1994 & 1995 & 1996 & 1997 & \begin{tabular}{|l|l|}
1998 & 1999 \\
\end{tabular} & 2000 \\
\hline $\begin{array}{l}\text { Politique budgétaire } \\
\text { (en \% du PIB) }\end{array}$ & $\cdot$ & \multicolumn{2}{|c|}{ expansion } & rest. & \multicolumn{2}{|c|}{ expansion } & \multicolumn{2}{|c|}{ restriction } & exp. \\
\hline $\begin{array}{l}\text { Dette publique } \\
\text { (en \% du PIB) }\end{array}$ & - & \multicolumn{3}{|c|}{ expansion } & rest. & exp. & rest. & stabilisation & rest. \\
\hline $\begin{array}{l}\text { Crédits à l'économie } \\
\text { (évolution annuelle en \%) }\end{array}$ & $\cdot$ & \multicolumn{2}{|c|}{ expansion } & \multicolumn{2}{|c|}{ restriction } & \multicolumn{2}{|c|}{$\begin{array}{c}\text { forte } \\
\text { expansion }\end{array}$} & $\begin{array}{c}\text { forte } \\
\text { restriction }\end{array}$ & - \\
\hline $\begin{array}{l}\text { Taux d'intérêt à l'emprunt } \\
(1 \text { an) }\end{array}$ & - & \multicolumn{3}{|c|}{ hausse } & b. & $\begin{array}{c}\text { forte } \\
\text { h. }\end{array}$ & \multicolumn{2}{|c|}{ stabilisation } & - \\
\hline$M_{3}$ & - & \multicolumn{3}{|c|}{ stabilisation } & rest. & exp. & \multicolumn{3}{|c|}{ restriction } \\
\hline $\begin{array}{l}\text { Dette externe } \\
\text { (en \% du PIB) }\end{array}$ & \multicolumn{2}{|c|}{ expansion } & \multicolumn{3}{|c|}{ restriction } & exp. & \multicolumn{3}{|c|}{ restriction } \\
\hline $\begin{array}{l}\text { Taux de change } \\
\text { (monn. nat./US\$) } \\
\text { (moyenne annuelle) }\end{array}$ & - & & & & & hausse & & & \\
\hline
\end{tabular}




\begin{tabular}{|c|c|c|c|c|c|c|c|c|c|c|}
\hline $\begin{array}{l}\text { Indicateurs } \\
\text { macroéconomiques }\end{array}$ & 1991 & 1992 & 2993 & 1994 & 1995 & 1996 & 1997 & 1998 & 1999 & 2000 \\
\hline $\begin{array}{l}\text { PIB réel/tête } \\
\text { (évolution annuelle en \%) }\end{array}$ & \multicolumn{3}{|c|}{ baisse } & \multicolumn{2}{|c|}{ hausse } & \multicolumn{3}{|c|}{ baisse } & \multicolumn{2}{|c|}{ hausse } \\
\hline $\begin{array}{l}\text { Investissements bruts } \\
\text { (en \% du PIB) }\end{array}$ & \multicolumn{4}{|c|}{ baisse } & h. & b. & \multicolumn{3}{|c|}{$\begin{array}{c}\text { hausse et } \\
\text { stabilisation }\end{array}$} & - \\
\hline Chômage & \multicolumn{3}{|c|}{ hausse } & \multicolumn{5}{|c|}{ baisse et stabilisation } & h. & - \\
\hline Inflation & \multicolumn{3}{|c|}{ forte $h$. et $b$. } & h. & b. & \multicolumn{2}{|c|}{ forte hausse } & b. & \multicolumn{2}{|c|}{ hausse } \\
\hline $\begin{array}{l}\text { Déficit de la balance } \\
\text { commerciale } \\
\text { (en \% du PIB) }\end{array}$ & - & \multicolumn{3}{|c|}{ hausse } & \multicolumn{3}{|c|}{ excédent positif } & \multicolumn{2}{|c|}{ hausse } & - \\
\hline
\end{tabular}

\section{Roumanie}

\begin{tabular}{|c|c|c|c|c|c|c|c|c|c|c|}
\hline Politique économique & 1991 & 1992 & 1993 & 1994 & 1995 & 1996 & 1997 & 1998 & 1999 & 2000 \\
\hline $\begin{array}{l}\text { Politique budgétaire } \\
\text { (en \% du PIB) }\end{array}$ & - & exp. & rest. & \multicolumn{3}{|c|}{ expansion } & \multicolumn{3}{|c|}{ restriction } & exp. \\
\hline $\begin{array}{l}\text { Dette publique } \\
\text { (en \% du PIB) }\end{array}$ & - & - & - & - & rest. & \multicolumn{3}{|c|}{$\begin{array}{l}\text { expansion et } \\
\text { stabilisation }\end{array}$} & exp. & rest. \\
\hline $\begin{array}{l}\text { Crédits à l'économie } \\
\text { (évolution annuelle en \%) }\end{array}$ & - & \multicolumn{4}{|c|}{ expansion } & \multicolumn{2}{|c|}{ restriction } & exp. & $\begin{array}{l}\text { forte } \\
\text { rest. }\end{array}$ & - \\
\hline $\begin{array}{l}\text { Taux d'intérêt à l'emprunt } \\
(1 \text { an) }\end{array}$ & - & - & h. & \multicolumn{2}{|c|}{ baisse } & \multicolumn{4}{|c|}{ hausse } & - \\
\hline$M_{3}$ & - & \multicolumn{4}{|c|}{ baisse } & \multicolumn{4}{|c|}{ hausse et stabilisation } & - \\
\hline $\begin{array}{l}\text { Dette externe } \\
\text { (en \% du PIB) }\end{array}$ & \multicolumn{7}{|c|}{ expansion } & b. & exp. & - \\
\hline $\begin{array}{l}\text { Taux de change } \\
\text { (monn. nat./US\$) } \\
\text { (moyenne annuelle) }\end{array}$ & - & \multicolumn{9}{|c|}{ hausse } \\
\hline \multicolumn{11}{|l|}{$\begin{array}{l}\text { Indicateurs } \\
\text { macroéconomiques }\end{array}$} \\
\hline $\begin{array}{l}\text { PIB réel/tête } \\
\text { (évolution annuelle en \%) }\end{array}$ & \multicolumn{2}{|c|}{ baisse } & \multicolumn{4}{|c|}{ hausse } & \multicolumn{3}{|c|}{ baisse } & $\begin{array}{c}\text { légère } \\
\text { h. }\end{array}$ \\
\hline $\begin{array}{l}\text { Investissements bruts } \\
\text { (en \% du PIB) }\end{array}$ & \multicolumn{3}{|c|}{ hausse } & & baisse et & & \multicolumn{3}{|c|}{ baisse } & - \\
\hline Chômage & \multicolumn{5}{|c|}{ hausse } & \multicolumn{2}{|c|}{\begin{tabular}{|c|} 
baisse et \\
stabilisation
\end{tabular}} & \multicolumn{2}{|c|}{\begin{tabular}{|c|} 
hausse et \\
stabilisation
\end{tabular}} & - \\
\hline Inflation & \multicolumn{4}{|c|}{$\begin{array}{l}\text { forte hausse et tendance } \\
\text { à la baisse }\end{array}$} & \multicolumn{2}{|c|}{ baisse } & $\begin{array}{c}\text { forte } \\
\text { h. }\end{array}$ & \multicolumn{3}{|c|}{$\begin{array}{l}\text { baisse mais } \\
\text { élevée }\end{array}$} \\
\hline $\begin{array}{l}\text { Déficit de la balance } \\
\text { commerciale } \\
\text { (en \% du PIB) }\end{array}$ & - & \multicolumn{4}{|c|}{ stabilisation } & \multicolumn{5}{|c|}{ hausse } \\
\hline
\end{tabular}

Sources : A partir de données provenant de :

BERD, Transition report 2000.

Series NAR.

The Road to Stability and Prosperity in South Eastern Europe, BERD / The World Bank, 2000.

Ivanov E. - Kouneva M., Investment Guide for Southeast Europe, Bulgaria Economic Forum NGO,

September 2000.

Kotios (Aggelos), Petrakos (George), éds., Restructuring and Development in Southeastern Europe, Volos : South and East European Development Center, University of Thessaly Press, 2000. 


\section{CONCLUSION}

Il est indispensable de garder une cohérence entre l'évolution des finances publiques, la politique monétaire, les réformes et l'ajustement structurel des économies en transition, afin d'éviter toute remise en question de la politique adoptée. L'expérience de cette décennie montre en effet que dans les pays qui nous concernent, lors de chaque période d'incohérence entre l'un ou l'autre élément, les économies ont subi de sérieux déséquilibres macroéconomiques, la politique économique par les moyens qu'elle se propose (austérité budgétaire et monétaire et introduction massive de réformes) n'arrivant pas à créer les conditions nécessaires à l'atténuation des blocages dont l'origine se trouve (entre autres) dans les caractéristiques examinées dans la première section. Or la cause de l'échec des politiques de stabilisation économique et de leur incapacité à engager ces pays sur un sentier de croissance durable semble être la non prise en compte de ces particularités.

L'application des mêmes mesures rigides de politique macroéconomique et l'introduction des mêmes réformes dans tous les pays en transition ne semble pas garantir à tous le passage réussi à l'économie de marché. Un certain nombre de pays est pris dans un cercle vicieux où il y a interaction négative entre les traits particuliers de leurs économies et de leur organisation sociale d'un côté, et de la politique macroéconomique de l'autre, dicréditant l'efficacité de cette dernière. L'alternance des directions que cette dernière poursuit, restrictives ou expansionnistes et même parfois contradictoires, et la persistance de ces traits ont bloqué le processus de la transiton dans une impasse où des périodes de crise succèdent à d'autres d'efforts d'assainissement économique. Bien sûr, l'utilisation d'instruments fiscaux et monétaires et l'introduction des réformes constituent l'essentiel de la transition, mais il est nécessaire de les ajuster judicieusement afin de réunir les conditions de réussite de l'entreprise. Cela peut concerner leur application totale, partielle ou progressive de sorte qu'ils soient cohérents et répondent surtout au degré de maturité des rapports socioéconomiques qui caractérisent chaque pays. 